\title{
Tracking Diverse Feelings and Activities Encourages Self-guided Holistic Behavior Change
}

\author{
Mina Khan \\ MIT Media Lab \\ Cambridge, MA, USA \\ minakhan01@gmail.com
}

\author{
Pattie Maes \\ MIT Media Lab \\ Cambridge, MA, USA \\ pattie@media.mit.edu
}

\begin{abstract}
Cognitive Behavioral Therapy highlights that people's thoughts, feelings, and behaviors are interconnected. Self-tracking, however, has mostly focused on a few emotions or activities. We created a mobile/web application to enable the users to track a broad range of daily activities and feelings in unison. In our 3-week study with 15 participants, the application improved the participants' selfawareness of their activities, feeling, and the correlation between their activities and feelings. Better self-awareness inspired personalized, self-guided, and holistic behavior change as the participants improved their self-care, daily planning and focus, and emotion regulation. Finally, the participants also developed a healthy and organized habit of self-reflection and even reflected on their holistic well-being and the meaning of having a 'good day'. Behavior change is usually treated as an external and isolated process but our research shows that tracking a broad range of activities and feelings can inspire self-guided holistic behavior change.
\end{abstract}

\section{CCS CONCEPTS}

\section{- Human-centered computing $\rightarrow$ User studies.}

\section{KEYWORDS}

self-awareness, activities, emotions, feelings, self-tracking, wellbeing, behavior change

\section{ACM Reference Format:}

Mina Khan and Pattie Maes. 2021. Tracking Diverse Feelings and Activities Encourages Self-guided Holistic Behavior Change. In Asian CHI Symposium 2021 (Asian CHI Symposium 2021), May 8-13, 2021, Yokohama, Japan. ACM, New York, NY, USA, 7 pages. https://doi.org/10.1145/3429360.3468190

\section{INTRODUCTION}

Self-tracking is commonly used to support self-awareness, selfmanagement, and behavior change. There are several automatic or manual self-tracking applications, e.g., for food, productivity, or physical activity tracking, or for chronic physical or mental health management. Most behavior change or self-tracking applications, however, focus on specific behavior change goals or on tracking only specific emotions or activities. Recent research points out that

Permission to make digital or hard copies of all or part of this work for personal or classroom use is granted without fee provided that copies are not made or distributed for profit or commercial advantage and that copies bear this notice and the full citation on the first page. Copyrights for components of this work owned by others than ACM must be honored. Abstracting with credit is permitted. To copy otherwise, or republish, to post on servers or to redistribute to lists, requires prior specific permission and/or a fee. Request permissions from permissions@acm.org.

Asian CHI Symposium 2021, May 8-13, 2021, Yokohama, Japan

(c) 2021 Association for Computing Machinery.

ACM ISBN 978-1-4503-8203-8/21/05 . \$ \$15.00

https://doi.org/10.1145/3429360.3468190 behavior change is not an isolated process as user behaviors and states are interconnected [40]. Also, Cognitive Behavioral Therapy (CBT) points out that people's feelings and behaviors are interconnected [42]. However, there are no investigations into tracking a broad range of possibly interconnected activities and feelings, without explicit behavior change goals.

We investigate the effects of self-tracking a broad range of activities and feelings, without explicit behavior change goals. We have 3 research questions: i. Effects of self-tracking a wide range of daily activities (without specific behavior change goals); ii. Effects of selftracking a wide range of daily emotions/feelings (without explicit behavior change goals); iii. Effects of tracking a broad range of activities and feelings in unison (without specific behavior change goals).

We created a mobile/web application for the users to self-report their daily activities and feelings. The users could report 8 activities using a 24-hour clock and 24 feelings (12 'positive'; 12 'negative') using a 5-point Likert scale. The users could also visualize their daily feelings and activities over time using two aligned graphs so they could correlate their feelings and activities over time. Our 3-week free-living study with 15 participants showed that self-tracking diverse activities and feelings not only helped self-awareness and self-reflection but also encouraged self-guided self-change. The participants not only changed different activities and regulated different emotions independently, but also correlated their activities and feelings so that different activities and feelings could influence each other. The participants also reflected on their holistic wellbeing and the meaning of having a good day. Thus, self-reporting and self-tracking a broad range of activities and feelings encouraged holistic, interconnected, and personalized behavior change.

Health is "complete physical, mental, and social well-being and not merely the absence of disease" [36]. We contribute a framework for self-tracking a broad range of feelings and activities and our study shows that self-tracking diverse and interconnected activities and feelings encourages holistic, personalized, and self-guided selfcare and self-improvement via self-awareness and self-reflection. Self-guided behavior change is important as behavior change is an internal process [40] and intrinsic motivation is important for change [25]. Also, self-guided behavior change is key for user autonomy and authenticity [43], and is also the most common long-term real-world behavior change [6]. Also, contrary to the commonlyused disconnected and didactic approach to behavior change, selftracking diverse and interconnected activities and feelings enables a holistic holistic self-awareness and self-guided behavior change. 


\section{RELATED WORK}

Self-tracking is common in digital health and there has been work on different self-tracking models $[15,26]$, domains [14], techniques, and uses. Our work extends previous research by allowing users to self-track diverse activities and feelings together, which were not addressed in previously literature. Action and reflection are important stages in self-tracking [26, 27] and our study shows that self-tracking diverse activities and feelings, without explicit behavior change goals, encourages self-guided and holistic selfawareness, self-reflection, and self-change. We categorize previous self-tracking work based on their target domains [14], techniques, and uses, and situate our work in previous literature.

\subsection{Self-tracking and Self-reflection Techniques}

Self-tracking can be done using three techniques: i. self-report using diaries and experience sampling, e.g., for wellness management based on Cognitive-Behavioral Therapy [32], for food and calorie tracking [9], and for recording thoughts and experiences for mindfulness and self-reflection [4, 28]; ii. automated sensing, e.g., for promoting physical activity [8]; iii. combination of selfreport and automated sensing, e.g., for health related habits [18] and diverse well-being [5]. There are also different techniques for self-reflection applications, e.g., via games [39], visualizations [2, 7], and even via physical objects [22]. We use self-report and simple visualizations, but we extend the current self-tracking work to include self-reporting of diverse daily activities and feelings, which have not been considered in previous work.

\subsection{Self-tracking Domains}

We grouped self-tracking applications in five different domains: i. Physical health, e.g., food [29], physical activity [8], and sleep management [45]; ii. Chronic disease management, e.g., multiple sclerosis [3], diabetes [13], Parkinson's disease [34], and endometriosis [33]; iii. Mental health, e.g., for psychotherapy [31], behavior change [19], cardiovascular disease support [35], stress management [20], and mental illness monitoring [41]; iv. Miscellaneous specific goals, e.g., fertility [11] or productivity [24]; v. General well-being and personal informatics, e.g., Health Mashups [5]. Our work is in the general well-being and personal informatics domain and our in situ study investigated the effects of tracking diverse activities and feelings, which were previously not addressed together in the aforementioned domains. Our study shows that tracking diverse activities and feelings encourages holistic, self-guided, and personalized behavior change.

\subsection{Self-tracking Uses}

There are five different uses of self-tracking: i. Detection, e.g., of mental illness symptoms [41]; ii. Forecasting, e.g., of blood glucose levels [13]; iii. Self-reflection, e.g., on bodily aspects of experiences and emotion [28], diabetes self-care [30], and overall wellbeing [5]; iv. Interventions, e.g., for chronic illnesses [37], emotion regulation [10], general psychological flexibility and well-being using Acceptance and Commitment Therapy [1], and physical activity support [8]; v. Self-experimentation, e.g., for sleep and [12] and individualized food triggers [21]. We extend the self-reflection and self-experimentation work by allowing the users to self-report diverse activities and feelings in unison, without explicit behavior change goals or interventions and without automatic user state prediction or detection.

\section{DESIGN AND IMPLEMENTATION}

We created a mobile/web application for tracking overall user feelings and activities. We share below our design guidelines, activities and feelings categories, and implementation and interface details.

\subsection{Design Guidelines}

Cognitive Behavior Therapy (CBT) highlights the connection between people's feelings and behaviors [42]. Loosely inspired by CBT, we decided to enable people to self-report and self-track their activities and feelings in unison. Moreover, since behavior change is interconnected and holistic [40] and since self-guided change is the most common long-term real-world behavior change, we did not set any specific self-tracking or behavior change goals for the users. Instead, we decided to allow people to track a wide variety of feelings and activities throughout the day. Since the state-of-the-art sensors cannot accurately detect a wide range of user activities and feelings, we decided to rely on self-report. However, in order to minimize the self-reporting effort for the user and to standardize user input, we decided to collect minimal and standardized user input, i.e., not lengthy and open-ended diaries. Also, we decided to minimize the frequency of user input, i.e., not expect frequent interactions throughout the day like in experience sampling, but only around once a day. Thus, our goal was to enable users to track diverse activities and feelings, without specific self-tracking or behavior change goals, and without lengthy or frequent user interactions per day.

\subsection{Activities and Feelings Categories}

We shared below the activity and feeling categories and their respective self-tracking interface details.

Activity Categories: We decided seven activity categories, loosely inspired by the Healthy Minds Platter [44], which recommends "Focus Time", "Connecting Time", "Play Time", "Physical Time", "Time In", "Down Time", and "Sleep Time" for supporting human well-being: i. Primary Work; ii. Secondary Work, e.g., emails and meetings; iii. Exercise; iv. Sleep; v. Personal time including downtime (e.g., watching Netflix), time-in (e.g. meditating), or playtime (e.g. playing computer games); vi. Social time, i.e., time with others; vii. Errands, chores, and miscellaneous. The users could choose their interpretation of different categories to give them flexibility over classifying different activities.

Feeling CAtegories: We decided to allow users to track positive and negative feelings as positive emotions can broaden the user's momentary perspective and long-term well-being [16], whereas negative emotions are also key for well-being [23]. Common lists like Ekman's six basic emotions, Plutchik's wheel of emotions [38], or PANAS test [46] did not include common user experiences like focused, productive, tired, etc. Thus, we decided to create our own list of commonly-experienced positive and negative feelings using expert and peer review (1 experts, 5 users). We created four categories of feelings (Heart, Mind, Body, and Spirit), each with a set of 
3 positive and negative emotions - i. Body: Energetic/Tired, Confident/Insecure, and Relaxed/Stressed; ii. Heart: Happy/Sad, Peaceful/Anxious, and Hopeful/Hopeless; iii. Mind: Focused/Chaotic, Inspired/Unchallenged, and Effective/Frustrated; iv. Spirit, i.e. feel towards others: Grateful/Disappointed, Extroverted/Withdrawn, and Empathetic/Carefree.

\subsection{Interface and Implementation}

We share details about our labeling interface and implementation below.

LABELING INTERFACE: We decided to use a 24-hour clock where the users could indicate their hourly activities. The user could only select one type of activity per hour and there was no overlap allowed for the sake of simplicity. Moreover, we use a five-point Likert scale to allow users to indicate the average intensity of each of their feelings per day. The default Likert value for each feeling is zero and the user is not required to input any of the values.

Implementation: We used Google's Polymer and Progressive Web Apps to create an application that could be installed on mobile phones like native applications and also works on desktops and laptops as a web application. In addition to activity and feeling input for each day, the app also simultaneously shows the activities and feelings over time so the users could compare the changes in their activities and feelings over time and also the correlations between them. Figure 1 shows the activities and feelings labeling interface for each day and also activities and feelings over time.

\section{STUDY DESIGN}

We conducted a 3-week study, $\mathrm{N}=15$ participants, to investigate the effects of self-tracking diverse activities and feelings.

Guidelines: We decided to take a free-living and phenomenological approach to evaluate user experiences [17]. The participants did not receive any financial compensation and were instructed to use the application if and whenever they wanted to. We also decided not focus on a specific target audience, e.g., users interested in a specific behavior change or with a specific healthy condition. Instead, we recruited "everyday" users, without any inclusion or exclusion criteria.

PARTICIPANTS: We sent a recruitment email for a 'self-tracking study' to our department email list, without any inclusion or exclusion criteria. We received interest from 15 people (10 females, 5 males; $\mu=27.6$ years, $\sigma=3.4$ years; 3 artists, 4 engineers, 5 students, and 3 staff members) and we recruited all of them via convenience sampling.

MEASURES: Each week the participants received a non-compulsory survey form with an open-ended question: "How was your experience with the application? How was it helpful or unhelpful? Anything else you would like to add?". At the end of the 3-week study, we scheduled an exit interview with all the participants. We also anonymously tracked the days on which the participants used the application to track activities and/or feelings.

\section{RESULTS}

We performed thematic analysis on the qualitative responses from the survey and summarize the results into three themes below -
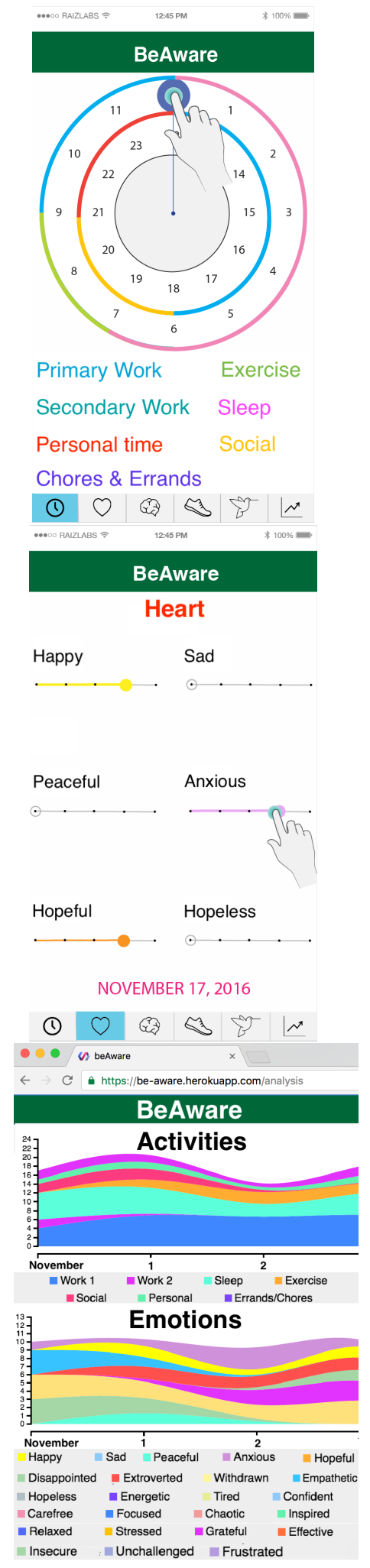

Figure 1: Interface for (a) self-reporting activities per day, (b) self-reporting feelings per day, and (c) tracking both over time 
self-care, self-awareness, and self-reflection. We also report on app usage and user's design suggestions.

\subsection{Self-awareness}

10 participants mentioned being more aware of their activities, feelings, and the interconnections between the two.

FEeLINGs: Two participants mentioned that they realized that they have been anxious. Another participant mentioned noticing emotions that they had not noticed before, "Using the app made me more aware of feelings and aspects of my life that I hadn't really thought about previously...Using the app made me pay attention to these emotions for the first time. Usually I never feel or think of hopelessness, but I noticed I felt exactly that".

Activities: Two participants mentioned that they realized that needed more sleep, and one participant mentioned that they realized that they had been spending too much social time. One participant mentioned that they realized that they "have not been had too much personal time" and another participant mentioned that they realized that they multitask too much, "I learned I am NOT mindful at all by default. I forget a lot of things. Entering my activities on the clock (1 activity per hour) made me realize that I multi-task too much".

Correlations between Activities and Feelings: The participants reported understanding the relationships between their activities and feelings, e.g. "I'm happier when I sleep more" and "social situations were stressing me out".

\subsection{Self-change}

A total of 11 participants mentioned changing their activities, regulating their emotions, and better planning their day.

ChANGing ACTIVITIEs: 5 participants mentioned changing their behaviors to take better care of themselves. Here are three examples: "I started to take more walks as active meditations"; "I feel as if last week's tracking of emotions allowed me to realize that social situations were stressing me out. This weekend, I bailed on all of my social commitments and spent a lot of time to myself"; "I really took stock of my way of being and I felt I needed to be more happy. So I actually shifted (!!!) what I did in my day to do less work (!!!) and more personal (!!!!), which is something I would normally have NOT EVER DONE...I took a lot more time for myself to do nothing, but I bounce back with more happiness when I get back to work."

EMOTION REGULATION: 4 participants mentioned that self-tracking helped them constructively deal with their feelings, especially the negative ones. One of the participants mentioned: "it hit me hard and I had to look inside to help my mind to interpret what was going on in the exterior...This self-reflection has allowed me to develop more patience, continue to be compassionate and caring, and be optimistic in my core". Another participant mentioned that self-reflection "...led to more productive ways of dealing with negative happenings rather than just reacting and doing nothing about it."

IMPROVED FOCUS AND PLANNING: 2 participants noted that selftracking helped them become more organized, e.g. "When I successfully get to record my day with the app, I tend to make to-do list for the next day as well, which is pretty helpful to start the next day more intentionally", and "It has made me focus on one task at a time... the need to record what I do later, sometimes makes me focus on one task at a time...I appreciate the app help me focus more".

\subsection{Self-reflection}

5 participants mentioned self-reflection in their comments. We share the different types of responses below.

REFLECTION ON MEANING: 3 participants mentioned that selftracking activities and feelings encouraged them to reflect on the 'meaning' of having a good day. One participant shared that he asked himself “What does a successful day for me look like?' It led me to design a day in terms of activities for that day, in hopes of establishing good habits like reading an hour a day...I would wonder if having a successful day is truly important if I am still unhappy after a successful week. e.g. having read a book an hour a day for a week, why do I still feel unaccomplished or unhappy?...”.

HABIT OF SELF-REFLECTION: One participant pointed out that they have been more in tune with themselves without having to rely on the app: "...I realized that, despite forgetting to enter the information on the app to track it, I've been keeping track of my emotions... I noticed I am more attuned to my own thoughts and feelings, allowing myself more time to understand them. When lesspositive emotions like anxiety and bad mood kick in, I'm able to identify them a bit more quickly, and I am able to take deep breaths before making decisions. I think this new understanding of what happens within me has helped me address my ongoing sleep issues. Now, before going to bed and letting negative thoughts invade my mind, I take time to breathe deeply, calm my mind, and welcome happier, calmer thoughts."

HeALThy AND ORGANIZED SELF-REFLECTION: One participant mentioned that, "I realized that I used to self-reflect too much and was harsh on myself, but with the app, I was able to objectively and categorically reflect on my feelings and activities. Having a simple framework to self-reflect and self-analyze made self-reflection less stressful and more productive".

\subsection{App Usage and Design Suggestions}

The participants used the application on an average 8.9 days (43\%) out of the 21 days. The average number of users per day was 8.75 (Week 1), 5.5 (Week 2), 2 (Week 3), and 5.8 (All 3 weeks). The usage declined over time and varied for different participants as shown in Figure 2. The participants did not explicitly mention anything explicitly unhelpful about the application. Four participants (1 in Week 1, 2 in Week 2, 1 in Week 3) mentioned that they forgot to track using the application during Week 3 , and three of those four mentioned that they would like automatic tracking and reminders to track and self-reflect. Also, in Week 3, two participants mentioned that they developed a "habit of self-reflection" and did not go to the app to record their feelings and activities. Also, six participants mentioned not needing self-tracking every day and did it as they "remembered" and "got time" every few days. Three participants also mentioned that they would like reminders to remind them of their desired behavior change. None mentioned any problems with the app.

\section{DISCUSSION}

Our study investigated the effects of self-tracking a broad range of daily user activities and feelings. We discuss the key findings, limitations, and design recommendations from our study below. 

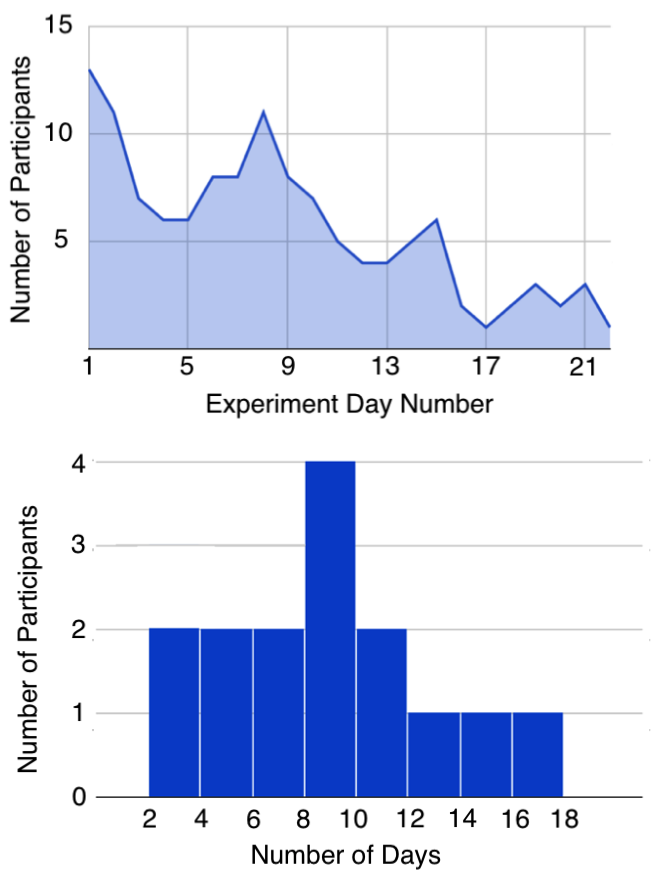

Figure 2: Statistics for app usage over time (left) and number of days for which different participants used the application (right)

\subsection{Key Findings}

We note five key findings from our study: i. Detailed activity tracking allows the users to not only reflect on their days but also to plan them better and make any changes if needed; ii. Detailed tracking of feelings/emotions allows users to be mindful of their emotions and self-regulate their feelings/emotions accordingly; iii. Simultaneously tracking activities and feelings enables the users to understand the correlation between their activities and feelings so that they can change one or more of their activities or feelings to guide the others; iv. Having a framework for self-tracking allows users to develop a healthy and organized habit of self-reflection, at least in the short-term; v. Overall self-tracking of a broad range of feelings and activities enables a bigger picture and personalized view of well-being, including purpose and meaning of different things, e.g., reading a book over more 'productive' work.

Overall, our study showed that self-tracking a wide range of activities and feelings not only enabled self-awareness about activities, feelings, and the interconnections between activities and feelings, but also encouraged and allowed the users to change different activities and feelings and even change an activity to change a feeling and vice versa. The users also reflected on their holistic well-being, including the meaning of having a 'good day'. Self-guided, multiple, intrinsically-motivated, and interconnected change is important as self-guided is the most common long-term and maintained realworld health behavior change [6]. Also, intrinsic motivation is key for behavior change [25]. Finally, recent research indicates that behavior change is an internal, multiple, and interconnected process [40].
Health is not the absence of illness, but a state of mental, physical, and emotional well-being. Moreover, self-tracking is not just about adding more user data but also about empowering users and enabling more autonomy and authenticity with respect to personal information and its uses [43]. Our work highlights that tracking diverse activities and feelings enables not only helpful self-reflection and self-awareness but also encourages self-guided, intrinsicallymotivated, and holistic behavior change for well-being, including meta-reflections, e.g., 'meaning'/'purpose' of a good day/life.

\subsection{Limitations}

As shown in the application usage results, the application usage fell over time. One of the reasons for the decline in app usage could be that the users developed a habit of self-reflection and did not have to rely on the application for self-reflection, which was mentioned by the participants. Also, it is difficult to take out time for and/or remember to self-report, especially every day. Four of our participants also requested automatic tracking. Thus, in order to reduce the burden on the users, we recommend automatic tracking of the aforementioned broad range of activities and feelings. While exact sensing of some feelings may be difficult, it may be possible to infer some of the feelings from user behavior, especially using some initial guidance from the users to learn personalized behavior patterns.

Moreover, even though self-tracking encourages self-guided behavior change, it may be effective for behavior change goal-setting but further studies need to investigate the objective effects on behavior change. Self-tracking may need to be augmented with other behavior change support, e.g., interventions or other persuasive techniques, for sustainable behavior change. In fact, three of our participants requested reminders for behavior change support.

Thus, self-tracking may be a good initial motivator, but may need to be augmented with automated sensing and other behavior change support strategies for effective long-term behavior change. None, tracking a broad range of activities and feelings helps initiate self-guided and holistic behavior change, unlike the commonly used externally-guided and isolated behavior change for only certain non-personalized activities/feelings.

\subsection{Design Recommendations}

Behavior change and behavior tracking technologies often focus on setting specific goals for the users and/or on tracking a narrow set of activities and physiological states, especially using automated sensors. We have three design recommendations from our study: i. Help users jointly track and correlate multiple activities and feelings to highlight the relationship between their activities and feelings; ii. Enable self-reflection without explicit behavior change goals to encourage the users to self-determine their personalized behavior change goals and techniques, especially since self-guided behavior change is the most common long-term real-world behavior change [6] and because intrinsic motivation is helpful for behavior change [25]; iii. Enable self-report, especially of diverse activities and feelings, because self-report can encourage self-reflection as the user is reporting the data themselves and also because the users can also report more diverse activities and feelings than can be automatically tracked by state-of-the-art sensors. 


\section{CONCLUSION}

Cognitive Behavioral Therapy highlights that people's feelings and behaviors are interconnected. There are a lot of applications for tracking user activities and emotions/feelings, but there are none for tracking a wide range of activities and feelings in unison. We investigated the effects of self-tracking a wide range of user activities and feelings in unison, without explicit behavior change goals. Our mobile/web app allowed the users to self-report 8 different daily activities (on a 24-hour clock) and 24 different daily feelings (using a 5-point Likert scale). The users could also visualize their feelings and activities over time using two aligned graphs, one for all activities and one for all feelings.

Our study showed that self-tracking activities and feelings improved participants' self-awareness, self-reflection, emotion regulation, daily planning, and focus. The participants mentioned developing a structured habit of self-reflection and used the correlation between their activities and feelings to guide changes in their activities, feelings, and/or both. The broad range of activities and feelings even inspired the participants to think about the 'meaning' of a good day/life.

Thus, self-tracking a broad range of activities and feelings in unison enabled a holistic and interconnected view of personal wellbeing and inspired self-guided and holistic self-change via selfawareness and self-reflection. Our research can further guide future self-tracking technologies to enable holistic self-tracking of daily activities and feelings to enable personalized self-awareness, selfreflection, and self-change, especially without explicit behavior change goals.

\section{REFERENCES}

[1] Aino Ahtinen, Pasi Välkkynen, Elina Mattila, Kirsikka Kaipainen, Miikka Ermes, Essi Sairanen, Tero Myllymäki, and Raimo Lappalainen. 2012. Oiva - A mobile phone intervention for psychological flexibility and wellbeing. In Designing for Wellness and Behavior Change workshop proceedings. Association for Computing Machinery ACM. https://cris.vtt.fi/en/publications/oiva-a-mobile-phoneintervention-for-psychological-flexibility-an

[2] Bon Adriel Aseniero, Charles Perin, Wesley Willett, Anthony Tang, and Sheelagh Carpendale. 2020. Activity River: Visualizing Planned and Logged Personal Activities for Reflection. In Proceedings of the International Conference on Advanced Visual Interfaces. 1-9.

[3] Amid Ayobi, Paul Marshall, and Anna L. Cox. 2020. Trackly: A Customisable and Pictorial Self-Tracking App to Support Agency in Multiple Sclerosis SelfCare. In Proceedings of the 2020 CHI Conference on Human Factors in Computing Systems (CHI '20). Association for Computing Machinery, New York, NY, USA, 1-15. https://doi.org/10.1145/3313831.3376809

[4] Amid Ayobi, Tobias Sonne, Paul Marshall, and Anna L. Cox. 2018. Flexible and Mindful Self-Tracking: Design Implications from Paper Bullet Journals. In Proceedings of the 2018 CHI Conference on Human Factors in Computing Systems (CHI '18). Association for Computing Machinery, New York, NY, USA, 1-14. https://doi.org/10.1145/3173574.3173602

[5] Frank Bentley, Konrad Tollmar, Peter Stephenson, Laura Levy, Brian Jones, Scott Robertson, Ed Price, Richard Catrambone, and Jeff Wilson. 2013. Health Mashups Presenting statistical patterns between wellbeing data and context in natural language to promote behavior change. ACM Transactions on Computer-Human Interaction (TOCHI) 20, 5 (2013), 1-27.

[6] F Michler Bishop. 2018. Self-guided Change: The most common form of longterm, maintained health behavior change. Health psychology open 5, 1 (2018), 2055102917751576.

[7] Sunny Consolvo, Predrag Klasnja, David W. McDonald, Daniel Avrahami, Jon Froehlich, Louis LeGrand, Ryan Libby, Keith Mosher, and James A. Landay. 2008. Flowers or a robot army? encouraging awareness \&amp; activity with personal, mobile displays. In Proceedings of the 10th international conference on Ubiquitous computing (UbiComp '08). Association for Computing Machinery, New York, NY, USA, 54-63. https://doi.org/10.1145/1409635.1409644

[8] Sunny Consolvo, David W. McDonald, Tammy Toscos, Mike Y. Chen, Jon Froehlich, Beverly Harrison, Predrag Klasnja, Anthony LaMarca, Louis LeGrand Ryan Libby, Ian Smith, and James A. Landay. 2008. Activity sensing in the wild: a field trial of ubifit garden. In Proceedings of the SIGCHI Conference on Human Factors in Computing Systems (CHI '08). Association for Computing Machinery, New York, NY, USA, 1797-1806. https://doi.org/10.1145/1357054.1357335

[9] Felicia Cordeiro, Elizabeth Bales, Erin Cherry, and James Fogarty. 2015. Rethinking the Mobile Food Journal: Exploring Opportunities for Lightweight PhotoBased Capture. In Proceedings of the 33rd Annual ACM Conference on Human Factors in Computing Systems (CHI '15). Association for Computing Machinery, New York, NY, USA, 3207-3216. https://doi.org/10.1145/2702123.2702154

[10] Jean Costa, Alexander T Adams, Malte F Jung, François Guimbretière, and Tanzeem Choudhury. 2016. EmotionCheck: leveraging bodily signals and false feedback to regulate our emotions. In Proceedings of the 2016 ACM International Joint Conference on Pervasive and Ubiquitous Computing. 758-769.

[11] Mayara Costa Figueiredo. 2020. Self-Tracking for Fertility Care: A Holistic Approach. In Extended Abstracts of the 2020 CHI Conference on Human Factors in Computing Systems (CHI EA '20). Association for Computing Machinery, New York, NY, USA, 1-9. https://doi.org/10.1145/3334480.3375029

[12] Nediyana Daskalova. 2018. Personalized Behavior-Powered Systems for Guiding Self-Experiments. In Extended Abstracts of the 2018 CHI Conference on Human Factors in Computing Systems (CHIEA '18). Association for Computing Machinery, New York, NY, USA, 1-4. https://doi.org/10.1145/3170427.3173017

[13] Pooja M. Desai, Elliot G. Mitchell, Maria L. Hwang, Matthew E. Levine, David J. Albers, and Lena Mamykina. 2019. Personal Health Oracle: Explorations of Personalized Predictions in Diabetes Self-Management. In Proceedings of the 2019 CHI Conference on Human Factors in Computing Systems (CHI '19). Association for Computing Machinery, New York, NY, USA, 1-13. https://doi.org/10.1145/ 3290605.3300600

[14] Daniel A Epstein, Clara Caldeira, Mayara Costa Figueiredo, Xi Lu, Lucas M Silva, Lucretia Williams, Jong Ho Lee, Qingyang Li, Simran Ahuja, Qiuer Chen, et al. 2020. Mapping and Taking Stock of the Personal Informatics Literature. Proceedings of the ACM on Interactive, Mobile, Wearable and Ubiquitous Technologies 4, 4 (2020), 1-38.

[15] Daniel A Epstein, An Ping, James Fogarty, and Sean A Munson. 2015. A lived informatics model of personal informatics. In Proceedings of the 2015 ACM International foint Conference on Pervasive and Ubiquitous Computing. 731-742.

[16] Barbara L Fredrickson and Thomas Joiner. 2002. Positive emotions trigger upward spirals toward emotional well-being. Psychological science 13, 2 (2002), 172-175.

[17] Eric B Hekler, Predrag Klasnja, Jon E Froehlich, and Matthew P Buman. 2013. Mind the Theoretical Gap: Interpreting, Using, and Developing Behavioral Theory in HCI Research. In Proceedings of the SIGCHI Conference on Human Factors in Computing Systems (Paris, France) (CHI '13). ACM, New York, NY, USA, 33073316.

[18] John Hicks, Nithya Ramanathan, Donnie Kim, Mohamad Monibi, Joshua Selsky, Mark Hansen, and Deborah Estrin. 2010. AndWellness: an open mobile system for activity and experience sampling. In Wireless Health 2010. 34-43.

[19] Victoria Hollis, Artie Konrad, and Steve Whittaker. 2015. Change of Heart: Emotion Tracking to Promote Behavior Change. In Proceedings of the 33rd Annual ACM Conference on Human Factors in Computing Systems (CHI '15). Association for Computing Machinery, New York, NY, USA, 2643-2652. https://doi.org/10. $1145 / 2702123.2702196$

[20] Eunkyung Jo, Austin L. Toombs, Colin M. Gray, and Hwajung Hong. 2020. Understanding Parenting Stress through Co-designed Self-Trackers. In Proceedings of the 2020 CHI Conference on Human Factors in Computing Systems (CHI '20). Association for Computing Machinery, New York, NY, USA, 1-13. https://doi.org/10.1145/3313831.3376359

[21] Ravi Karkar, Jessica Schroeder, Daniel A. Epstein, Laura R. Pina, Jeffrey Scofield, James Fogarty, Julie A. Kientz, Sean A. Munson, Roger Vilardaga, and Jasmine Zia. 2017. TummyTrials: A Feasibility Study of Using Self-Experimentation to Detect Individualized Food Triggers. In Proceedings of the 2017 CHI Conference on Human Factors in Computing Systems (CHI '17). Association for Computing Machinery, New York, NY, USA, 6850-6863. https://doi.org/10.1145/3025453.3025480

[22] Maria Karyda, Merja Ryöppy, Jacob Buur, and Andrés Lucero. 2020. Imagining Data-Objects for Reflective Self-Tracking. In Proceedings of the 2020 CHI Conference on Human Factors in Computing Systems (CHI '20). Association for Computing Machinery, New York, NY, USA, 1-12. https://doi.org/10.1145/3313831.3376844

[23] Todd Kashdan and Robert Biswas-Diener. 2014. The upside of your dark side: Why being your whole self-not just your" good" self-drives success and fulfillment. Penguin.

[24] Young-Ho Kim, Eun Kyoung Choe, Bongshin Lee, and Jinwook Seo. 2019. Understanding Personal Productivity: How Knowledge Workers Define, Evaluate, and Reflect on Their Productivity. In Proceedings of the 2019 CHI Conference on Human Factors in Computing Systems (CHI '19). Association for Computing Machinery, New York, NY, USA, 1-12. https://doi.org/10.1145/3290605.3300845

[25] Dominika Kwasnicka, Stephan U Dombrowski, Martin White, and Falko Sniehotta. 2016. Theoretical explanations for maintenance of behaviour change: a systematic review of behaviour theories. Health Psychol. Rev. 10, 3 (Sept. 2016), 277-296.

[26] Ian Li, Anind Dey, and Jodi Forlizzi. 2010. A stage-based model of personal informatics systems. In Proceedings of the SIGCHI conference on human factors in computing systems. 557-566. 
[27] Ian Li, Anind K Dey, and Jodi Forlizzi. 2011. Understanding my data, myself: supporting self-reflection with ubicomp technologies. In Proceedings of the 13th international conference on Ubiquitous computing. 405-414.

[28] Madelene Lindström, Anna Ståhl, Kristina Höök, Petra Sundström, Jarmo Laaksolathi, Marco Combetto, Alex Taylor, and Roberto Bresin. 2006. Affective diary: designing for bodily expressiveness and self-reflection. In $\mathrm{CHI}$ '06 Extended Abstracts on Human Factors in Computing Systems (CHI EA '06). Association for Computing Machinery, New York, NY, USA, 1037-1042. https: //doi.org/10.1145/1125451.1125649

[29] Yuhan Luo, Peiyi Liu, and Eun Kyoung Choe. 2019. Co-Designing Food Trackers with Dietitians: Identifying Design Opportunities for Food Tracker Customization In Proceedings of the 2019 CHI Conference on Human Factors in Computing Systems (CHI '19). Association for Computing Machinery, New York, NY, USA, 1-13. https://doi.org/10.1145/3290605.3300822

[30] Lena Mamykina, Elizabeth Mynatt, Patricia Davidson, and Daniel Greenblatt. 2008. MAHI: investigation of social scaffolding for reflective thinking in diabetes management. In Proceedings of the SIGCHI Conference on Human Factors in Computing Systems (CHI '08). Association for Computing Machinery, New York, NY, USA, 477-486. https://doi.org/10.1145/1357054.1357131

[31] Mark Matthews and Gavin Doherty. 2011. In the mood: engaging teenagers in psychotherapy using mobile phones. In Proceedings of the SIGCHI Conference on Human Factors in Computing Systems. 2947-2956.

[32] E. Mattila, J. Parkka, M. Hermersdorf, J. Kaasinen, J. Vainio, K. Samposalo, J. Merilahti, J. Kolari, M. Kulju, R. Lappalainen, and I. Korhonen. 2008. Mobile Diary for Wellness Management\&\#x2014;Results on Usage and Usability in Two User Studies. IEEE Transactions on Information Technology in Biomedicine 12, 4 (July 2008), 501-512. https://doi.org/10.1109/TITB.2007.908237

[33] Mollie McKillop, Lena Mamykina, and Noémie Elhadad. 2018. Designing in the Dark: Eliciting Self-tracking Dimensions for Understanding Enigmatic Disease. In Proceedings of the 2018 CHI Conference on Human Factors in Computing Systems (CHI '18). Association for Computing Machinery, New York, NY, USA, 1-15. https://doi.org/10.1145/3173574.3174139

[34] Sonali R. Mishra, Predrag Klasnja, John MacDuffie Woodburn, Eric B. Hekler, Larsson Omberg, Michael Kellen, and Lara Mangravite. 2019. Supporting Coping with Parkinson's Disease Through Self Tracking. In Proceedings of the 2019 CHI Conference on Human Factors in Computing Systems (CHI '19). Association for Computing Machinery, New York, NY, USA, 1-16. https://doi.org/10.1145/ 3290605.3300337

[35] Margaret E. Morris, Qusai Kathawala, Todd K. Leen, Ethan E. Gorenstein, Farzin Guilak, William DeLeeuw, and Michael Labhard. 2010. Mobile Therapy: Case Study Evaluations of a Cell Phone Application for Emotional Self-Awareness. fournal of Medical Internet Research 12, 2 (2010), e10. https://doi.org/10.2196/ jmir.1371 Company: Journal of Medical Internet Research Distributor: Journal of Medical Internet Research Institution: Journal of Medical Internet Research Label: Journal of Medical Internet Research Publisher: JMIR Publications Inc., Toronto, Canada.

[36] World Health Organization et al. 1948. Preamble to the Constitution of the World Health Organization as adopted by the International Health Conference, New York, 19-22 June, 1946; signed on 22 July 1946 by the representatives of 61 States (Official Records of the World Health Organization, no. 2, p. 100) and entered into force on 7 April 1948. http://www. who. int/governance/eb/who_constitution_en. pdf (1948).

[37] Tamara Peyton and Erika Poole. 2013. "My Mobile Health, My Mobile Life": methods for designing health interventions with adolescents. In CHI '13 Extended Abstracts on Human Factors in Computing Systems (CHI EA '13). Association for Computing Machinery, New York, NY, USA, 481-486. https://doi.org/10.1145/ 2468356.2468441

[38] Robert Plutchik. 2001. The Nature of Emotions: Human emotions have deep evolutionary roots, a fact that may explain their complexity and provide tools for clinical practice. American Scientist 89, 4 (2001), 344-350. https://www.jstor. org/stable/27857503 Publisher: Sigma Xi, The Scientific Research Society.

[39] Amon Rapp. 2018. Gamification for Self-Tracking: From World of Warcraft to the Design of Personal Informatics Systems. In Proceedings of the 2018 CHI Conference on Human Factors in Computing Systems (CHI '18). Association for Computing Machinery, New York, NY, USA, 1-15. https://doi.org/10.1145/3173574.3173654

[40] Amon Rapp, Maurizio Tirassa, and Lia Tirabeni. 2019. Rethinking Technologies for Behavior Change: A View from the Inside of Human Change. ACM Transactions on Computer-Human Interaction 26, 4 (June 2019), 22:1-22:30. https: //doi.org/10.1145/3318142

[41] Erik Reinertsen and Gari D Clifford. 2018. A review of physiological and behavioral monitoring with digital sensors for neuropsychiatric illnesses. Physiol. Meas. 39, 5 (May 2018), 05TR01.

[42] Barbara Olasov Rothbaum, Elizabeth A Meadows, Patricia Resick, and David W Foy. 2000. Cognitive-behavioral therapy. (2000).

[43] Tamar Sharon. 2017. Self-tracking for health and the quantified self: Rearticulating autonomy, solidarity, and authenticity in an age of personalized healthcare. Philosophy \& Technology 30, 1 (2017), 93-121.

[44] D Siegel. 2020. The Healthy Mind Platter. Viitattu 17 (2020), 2020.

45] Andrew Steptoe, Katie O’Donnell, Michael Marmot, and Jane Wardle. 2008. Positive affect, psychological well-being, and good sleep. fournal of Psychosomatic Research 64, 4 (April 2008), 409-415. https://doi.org/10.1016/j.jpsychores.2007. 11.008

[46] David Watson, Lee Anna Clark, and Auke Tellegen. 1988. Development and validation of brief measures of positive and negative affect: the PANAS scales. Journal of personality and social psychology 54, 6 (1988), 1063. 